\title{
Interactive Deep Learning for Detailed Seismic Interpretation of Structurally Complex Areas: A Campos Basin Case Study
}

\author{
Ana Krueger ${ }^{1}$, Julian Chenin ${ }^{1}$, Scotty Salamoff ${ }^{1}$, Benjamin Lartigue ${ }^{1}$, Paul Endresen ${ }^{1},{ }^{1}$ Bluware Corporation
}

\begin{abstract}
Copyright 2021, SBGf - Sociedade Brasileira de Geofísica
This paper was prepared for presentation during the $17^{\text {th }}$ International Congress of the Brazilian Geophysical Society held in Rio de Janeiro, Brazil, 8-11 November 2021.

Contents of this paper were reviewed by the Technical Committee of the $17^{\text {th }}$ International Congress of the Brazilian Geophysical Society and do not necessarily represent any position of the SBGf, its officers or members. Electronic reproduction or storage of any part of this paper for commercial purposes without the written consent of the Brazilian Geophysical Society is prohibited.
\end{abstract}

\begin{abstract}
The largest post-salt turbidite oil fields in the world are situated in the Campos Basin, whereas the largest pre-salt microbialite carbonate reservoirs oil fields in the world are situated in the Santos Basin. Salt tectonics (halokinetics) control the structural fabrics of the Campos Basin. Postsalt deepwater turbidite plays that were deposited synchronously to halokinesis and reservoir characterization are tied to the development of the salt mini-basins. Future offshore exploration will continue targeting these post-salt deepwater and ultra-deepwater mini-basins in addition to the pre-salt plays under the massive salt walls, which still remains an under-explored frontier. In this case study, we propose a new approach to deep learning that augments seismic interpretation and provides further detail to understand this complex play.
\end{abstract}

\section{Introduction}

The main producing deepwater oil fields in Campos Basin are in shelf-derived turbiditic sands encountered in the upper and lower continental slope from the Miocene to Upper Cretaceous. From their discovery period between 1985 to 1997 , these deepwater sands continue to produce in the Campos Basin (Guardado et al., 2000). Among these are the Marlim, Albacora, Roncador, Barracuda and Espadarte Fields. The oil and gas plays are mostly confined to stratigraphic features within the higher horst blocks. However, in deepwater, these are primarily turbidite plays. Campos Basin turbidite reservoirs are encountered in the upper and lower continental slope from the Miocene to Upper Cretaceous and in distal marine turbidite channel sands of the lower slope. Recoverable reserves for the giant Albacora, Marlin and Roncador Fields are expected to exceed $10 \mathrm{BBO}$ throughout their time span of production (Fainstein et al., 2019).

The structural framework of these deepwater plays is entirely controlled by salt tectonics. The salt fairway becomes progressively narrower towards the north (Bahia and Sergipe), and no salt is encountered in the equatorial transform margin basins (Fainstein et al., 2019). Syndepositional differential loading within the turbidite sequences were the main driving mechanism for salt evacuation. This caused the salt layers to thin and form tilted faulted blocks seaward dipping, where anticline features are formed in association with block bounding listric faults, resulting in turtle-back structures mainly involving the Albian carbonates and turbidite reservoirs within Paleogene to Neogene mini-basins (Krueger and Gilbert, 2007 and Krueger and Gilbert, 2009).

Lacustrine sediments of the syn-rift reached the oil generation window around the Eocene time period. Source rocks are separated from the main reservoirs by a salt layer, thus oil migration from source to reservoirs required migration pathways through salt windows (Mello et al., 1994). In regions where salt is absent, oil reached the younger reservoirs along faults related to salt movement.

The deepwater plays required substantial exploration investments, such that during the contemporary period after 1997 up to the first decade of the $21^{\text {st }}$ century, more than 200 exploratory wells have been drilled on water depths greater than $1 \mathrm{~km}$. New oil discoveries have been made in the Santos, Campos and Espírito Santo Basins (Fainstein et al., 2019). In the mature Campos Basin, a focus on detailed interpretation is key for creating detailed reservoir models, which are required for continued production and for the opportunity to add satellite opportunities.

With deep learning, it is now possible to add the level of detail needed to develop these plays, by mapping detailed salt surfaces and thousands of faults in a short period of time. To demonstrate the feasibility of this workflow, we conducted a case study within the northern region of the Campos Basin using a 3D seismic survey (Figure 1). The study area contains a plethora of faults and salt features, such as salt welds and overhangs, that are particularly challenging to map.

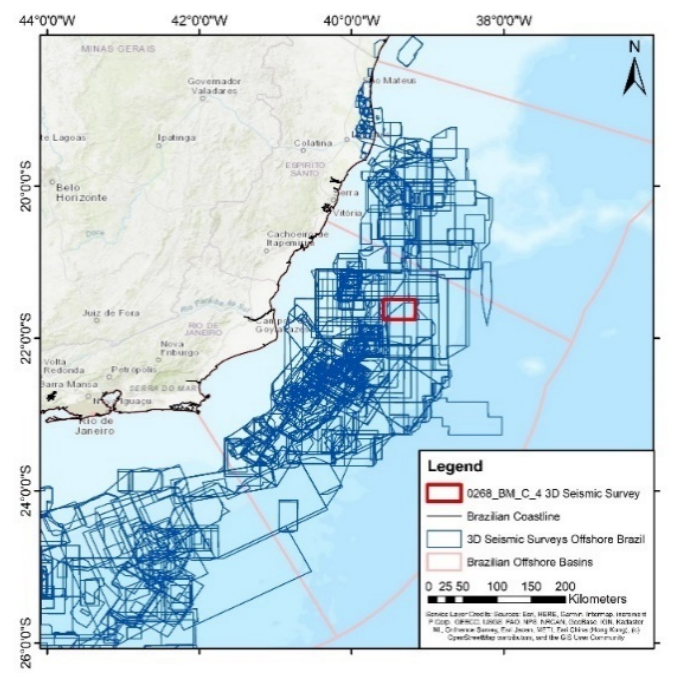

Figure 1 - Location of the $3 D$ seismic survey used in the case study of the Campos Basin. 


\section{Methodology}

Many recent studies (LeCun et al., 2015; Ronneberger et al., 2015; Bandura et al., 2018; Chopra and Marfurt, 2018; Chenin and Bedle, 2019) have highlighted the promising applications of machine learning for analyzing and interpretating geologic patterns. We apply a deep learning methodology which differs from current ones, due to the high level of interactivity it provides between the deep learning process and the interpreter. This process is referred to as interactive deep learning.

The key differences between traditional and interactive deep learning approaches are shown in Figure 2 . Traditional methods require significant data preparation before the network can be run, since TensorFlow records are static and cannot be modified on the fly. However, this unnecessary burden is avoided using our interactive methodology (Figure 2). Interactive deep learning is possible using random access and proprietary compression technology for seismic data.
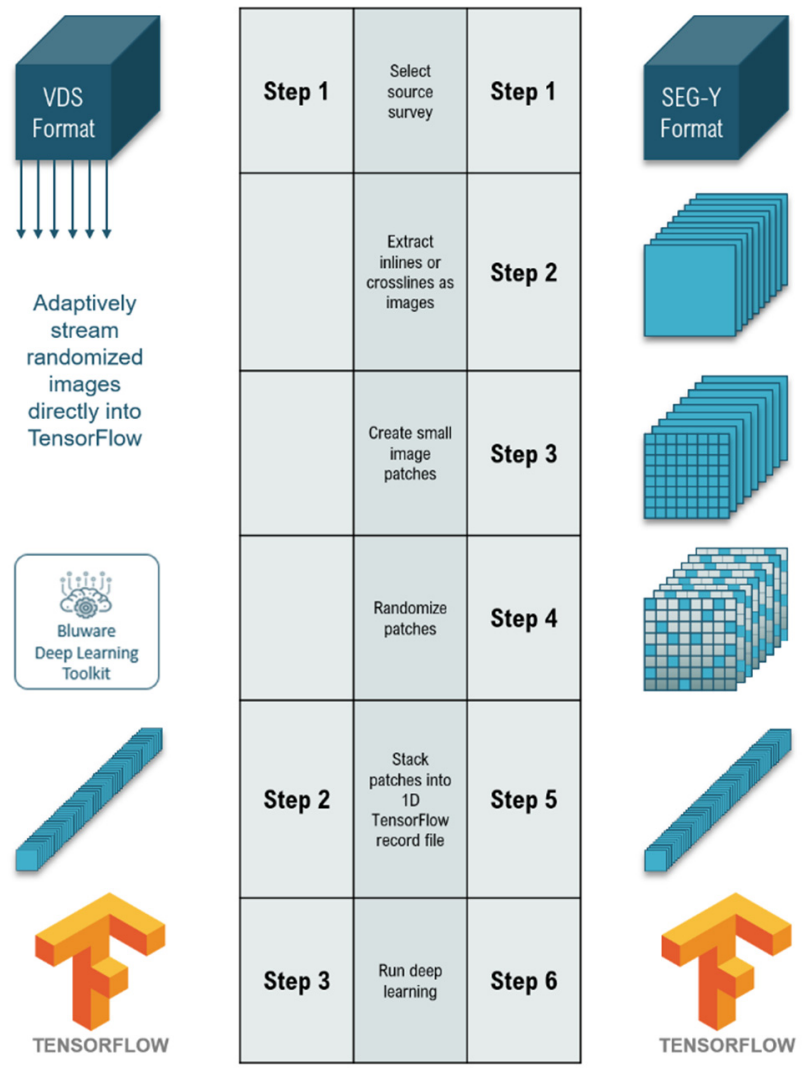

Figure 2 - Comparison of traditional and interactive deep learning technology for seismic interpretation. With traditional deep learning technology, the process starts with SEGY data. These patches are later created and randomized. With the interactive deep learning methodology, steps 2, 3, 4 and 5 are eliminated, thus accelerating the time to obtain a solution.

Deep learning is accelerated to enable interactive feedback by using random access to seismic data. Our methodology allows the geoscientist to label the data, train the network, and analyze the predictions (also referred to as inference) in real-time with different configurations. Therefore, the geoscientist does not rely on "black box" utilities to generate a high-quality result and can instead provide active feedback and reinforcement to the network while concurrently training it. The geoscientist can keep training until they achieve the optimal inference result. This method embeds quality control with training and interpretation, therefore removing the disadvantage of laborious and tedious quality control.

We present our custom deep learning approach that reduces the dimensions using "valid padding" between the features (seismic data) and the labels (interpretations created by the geoscientist). The reduction uses "valid padding", instead of "same padding", during the convolutions to maximize the amount of information into the Convolutional Neural Network (CNN) computation (Figure 3). While "same padding" has some advantages for designing networks easily, in our network it is disadvantageous, as it fills the convolutions with zeros, therefore omitting some of the data near the edges of the patches. Finally, our deep learning model was defined using a small number of layers and trainable weights to allow for rapid calculations and accurate results. The deep learning model can now be operated interactively, providing swift responses to interpreter input.

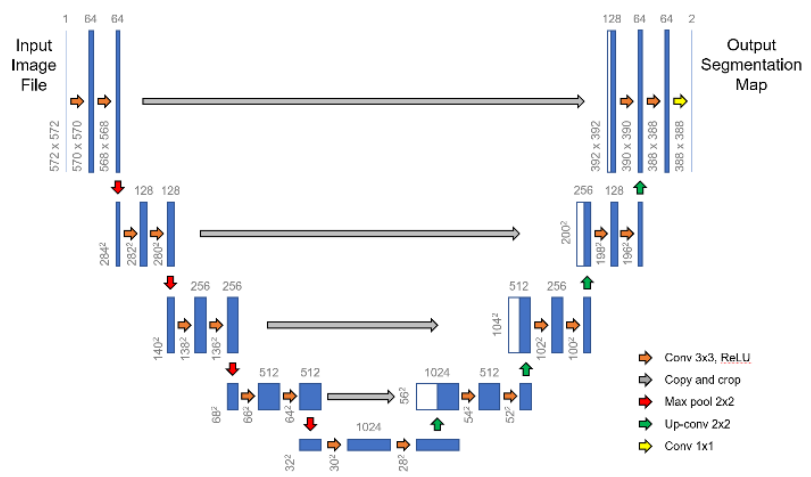

Figure 3 - Example of a CNN adapted from Ronneberger et al., 2015 consisting of a combination of different operations called "convolution" and "down-sampling/max pooling". The number and combinations of convolutions and max pooling operations within a CNN can vary for a plethora of reasons, mostly depending on the type of problem being solved. It is important to note that our method uses slightly different input and output sizes compared to this model.

\section{Interactive Deep Learning: Fault and Salt Labels}

The post-stack seismic survey used in this case study is composed of 1272 inlines and 3550 crosslines. For the fault deep learning network, we labeled 5 inlines and 10 crosslines (Figure 4A-B). As can be seen in Figure 4A-B, the labelled lines are not evenly distributed along the survey. The method used to select these lines was based on their unique representation of the fault geometries of interest. All faults that were mapped are either associated to salt evacuation features, or to folding associated to the formation of salt diapirs, salt walls, and turtle structures. 


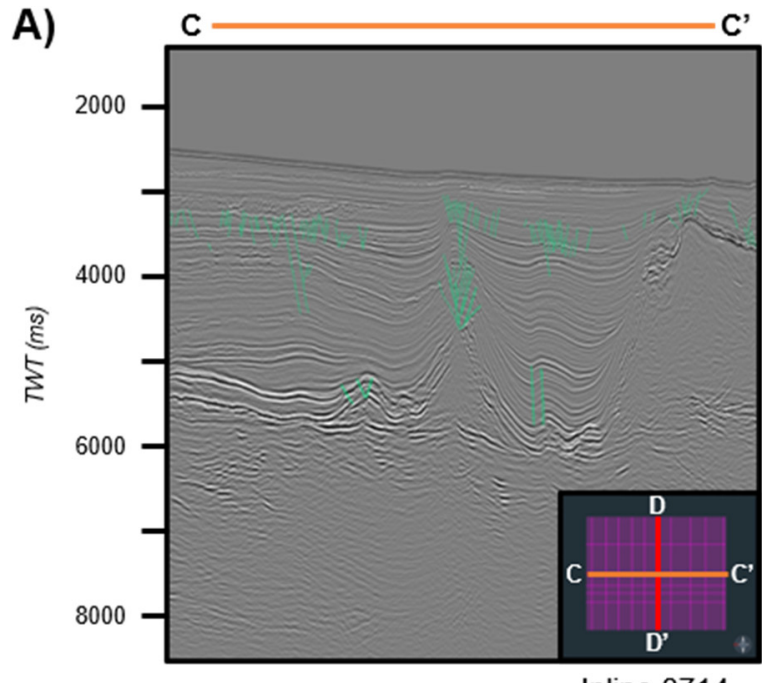

Inline 8714

B)

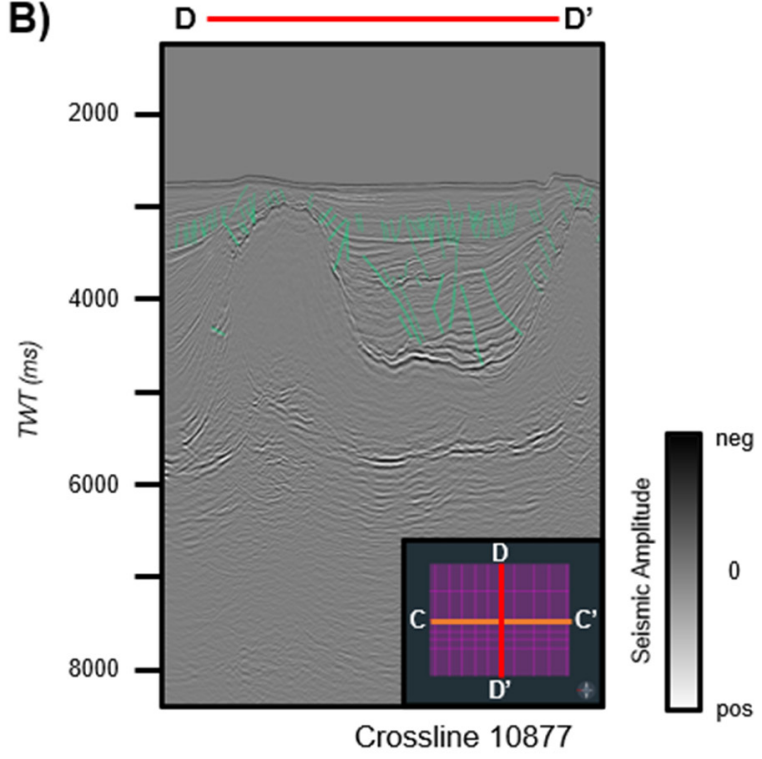

Figure 4 - Example of faults labelled on A) an inline and $B)$ a crossline. The pink lines on the $2 D$ map represent the other labelled lines used for training the fault network.

For the salt network, a total of 6 inlines and 3 crosslines were labelled (Figure 5A-B). Similar to the labelling workflows for the fault network, we labeled the lines that represented the variability of the seismic expression of the salt observed in the survey, as well as the varying signal to noise ratio. The labels included various salt structures such as salt pillows, salt walls, and salt welds.

\section{Results}

The fault network was trained for roughly 15 epochs (total time of about 11 minutes) whereas the salt network was trained for approximately 50 epochs (total time of about 37 minutes). Both networks were trained separately and from scratch until the inference exceeded an accuracy greater

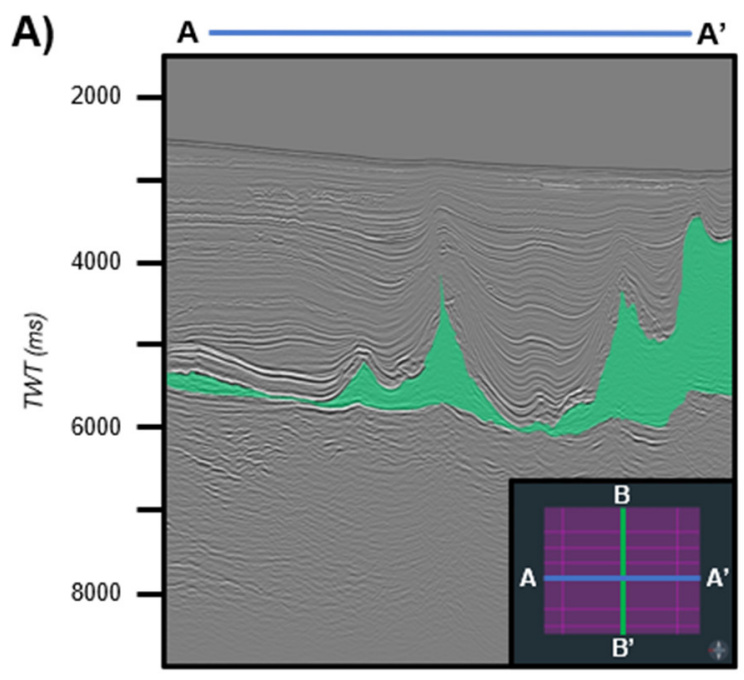

Inline 8650

B)

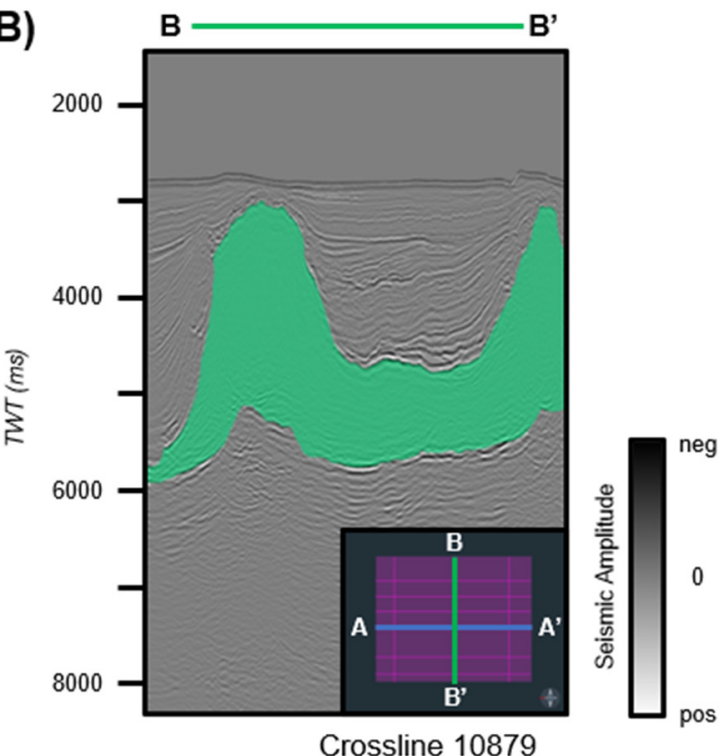

Figure 5 - Example of salt labelled $A$ ) on an inline and $B$ ) a crossline. The pink lines on the $2 D$ map represent the other labelled lines used for training the fault network.

than $90 \%$. Inference results are displayed in Figures $6 A-B$ and 7 terms of probability volumes for both faults and salt. Using very few lines, we were able to train both models, create detailed inference volumes, and output surfaces for salt features and thousands of faults within a day. The fault network was able to successfully infer both normal and reverse faults.

Furthermore, the salt network was able to predict the obvious salt diapirs and the more elusive salt welds. The inline on Figure 6A, highlights the highly rugose salt pillows with normal and reverse faults on top. The inference on the crossline shown in Figure 6B accurately captures the flower structure and the thin salt layer. Figure 7 highlights the relationship between the faults and salt in a time slice, 
where salt mini-basins are flanked by diapirs, and are highly faulted.

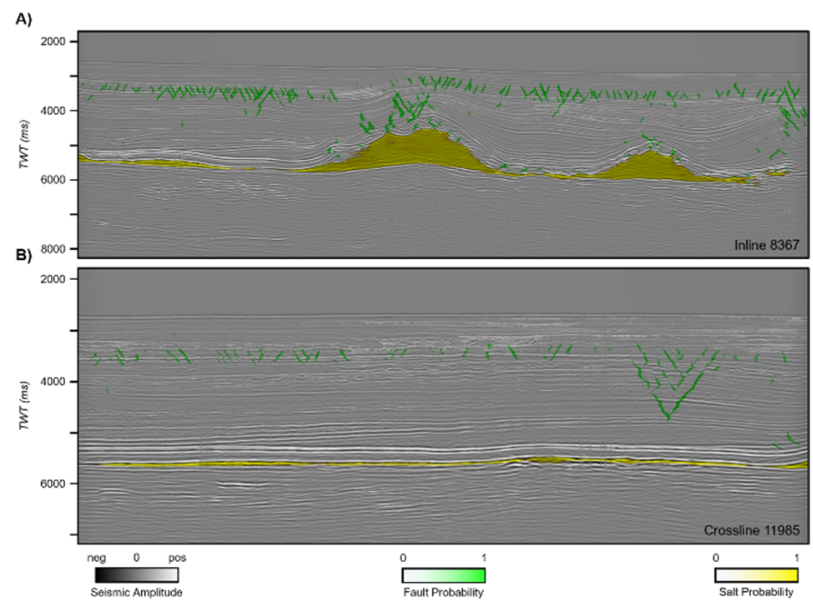

Figure 6 - Example of the inference displayed for both salt and faults simultaneously on A) an inline and B) a crossline.

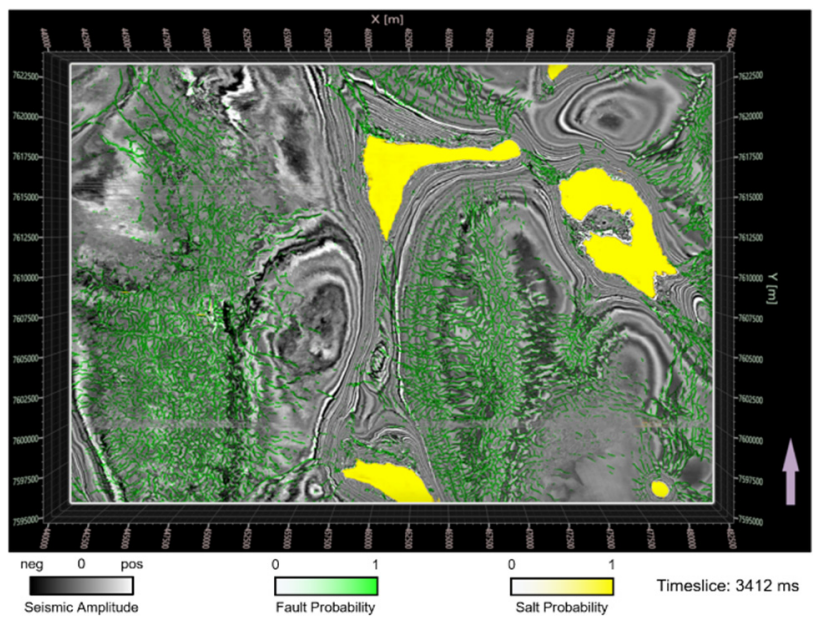

Figure 7 - Timeslice taken at 3412 ms displaying the predictions for both faults and salt. Note how the prediction was able to differentiate between salt and rafted sediment in the upper right corner.

Once satisfied with the fault and salt results, fault surfaces and salt geobodies was extracted from the deep learning networks' inferences using proprietary algorithms. Minimal post-processing, such as applying a gaussian smoothing filter of 4, was performed on the surfaces and geobodies. Figure 8 displays the final salt geobodies and fault surfaces generated from deep learning network predictions.

Throughout the dataset, both inferences were accurate and captured the true extent of salt tectonics and associated deformation. While some slight misclassifications are present within both networks, they were able to identify these geologic patterns in a similar manner as a seismic interpreter. Nevertheless, these complex geologic features identified by the deep learning network enables the geoscientist to gain further insights into the complex petroleum system of the Campos Basin.

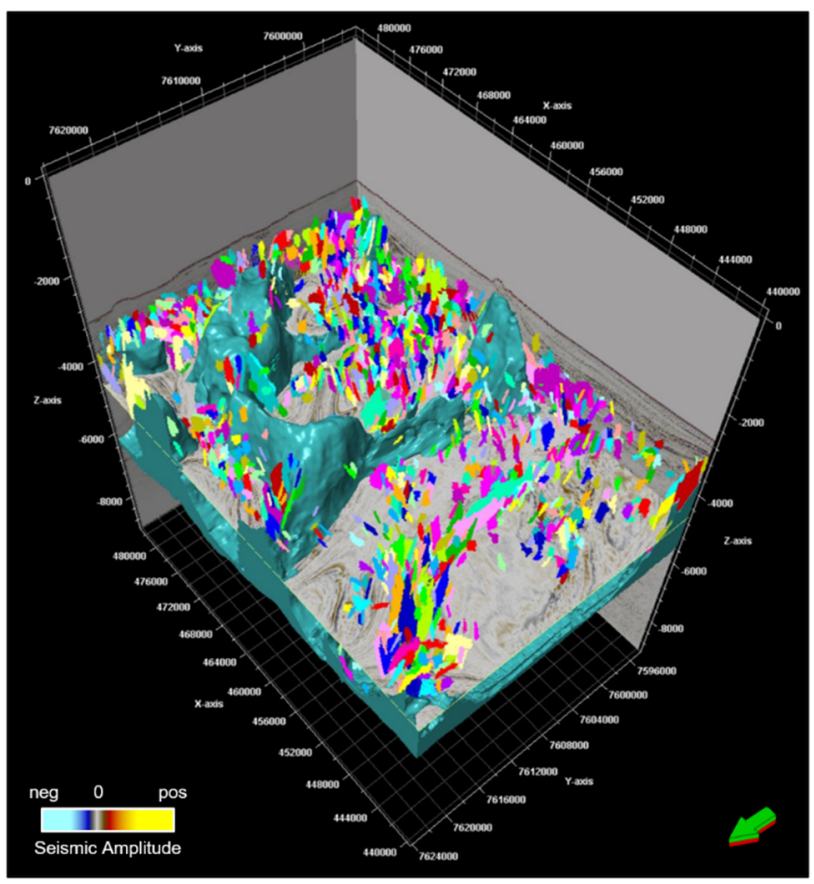

Figure $8-3 D$ seismic volume displayed together with the fault surfaces and the salt geobodies generated from their respective deep learning networks.

\section{Concluding Remarks}

Within a short period of time, we were able to use deep learning to map all faults and salt geobodies in a 3D seismic survey. We started with an untrained network and were able to create a detailed representation of the geology and petroleum system within the Campos Basin. With the ability to add this level of detail to the interpretation, geoscientists can now gain an unprecedented level of understanding of the petroleum system and reservoir models. The technology of interactive deep learning has the potential to optimize exploration and improve interpretations, while doing so in a fraction of the time and minimizing interpretation error.

\section{Acknowledgments}

We thank ANP (Agencia Nacional de Petroleo) Brazil for providing access to this seismic dataset. We also thank the team at Bluware for their helpful advice and feedback throughout this project.

This work was performed using InteractivAl, a commercial software product owned and marketed by the Bluware Corporation. 


\section{References}

Bandura, L., S. Halpert, and Z. Zhang, 2018, Machine Learning in the Interpreter's toolbox: unsupervised, supervised, and deep learning applications: 88th Annual SEG Expo, https://doi.org/10.1190/segam20182997015.1.

Chenin, J., and H. Bedle, 2020, Multi-attribute machine learning analysis for weak BSR detection in the Pegasus Basin, Offshore New Zealand: Marine Geophysical Research, 41, no. 4, 1-20, https://doi.org/10.1007/s11001020-09421-x.

Chopra, S., and K. J. Marfurt, 2018, Seismic facies classification using some unsupervised machine-learning methods: SEG Technical Program Expanded Abstracts 2018, 2056-2060, https://doi.org/10.1190/segam20182997356.1.

Chopra, S., and K. J. Marfurt, 2005, Seismic attributes-A historical perspective: Geophysics, 70 , no. 5, 3SO-28SO, https://doi.org/10.1190/1.2098670.

Fainstein, R., A. Krueger, and W. Ueipass Mohriak, 2019, Ultra-deepwater seismic plays offshore Brazil-Future drilling off Santos and Campos Basins: Interpretation, 7, no. 4, SH99-SH109, https://doi.org/10.1190/INT-2018$\underline{0251.1}$.

Guardado, L.R., Spadini, A.R., Brandão, J.S.L., and Mello, M.R., 2000. Petroleum System of the Campos Basin, Brazil. in M.R. Mello and B.J. Katz, eds., Petroleum systems of South Atlantic margins: AAPG Memoir 73, p. 317- 324

Krueger, A.C., and E. Gilbert, 2009, Deepwater FoldThrust Belts: Not All the Beasts Are Equal. AAPG Search and Discovery Article \#30085. AAPG International Conference, Cape Town, South Africa, 2009.

Krueger, A., and Gilbert E., 2007. Rethinking Controls on the Mechanics of Deepwater Thrusting-Interaction of Structure and Sedimentation. AAPG Search and Discovery Article \#90072. AAPG and AAPG European Region Conference, Athens, Greece, 2007.

LeCun, Y., Y. Bengio, and G. Hinton, 2015, Deep Learning: Nature, 521, no. 7553, 436-444, https://doi.org/10.1038/nature14539.

Mello, M.R., Mohriak, W.U., Koutsoukos, E.A.M., and Bacoccoli, G., 1994. Selected Petroleum Systems in Brazil. In: Magoon, L.B. and W.G. Dow, eds., 1994, The petroleum system -from source to trap: AAPG Memoir 60, p. $499-512$.

Ronneberger, O., P. Fischer, and T. Brox, 2015, U-net: Convolutional networks for biomedical image segmentation: International Conference on Medical Image Computing and Computer-Assisted Intervention 2015, 9351, 234-241, https://doi.org/10.1007/978-3-319-24574428. 